\title{
Quiste hidatídico hepático complicado con invasión a vena cava inferior y embolización de membranas hidatídicas a aurícula derecha. Caso clínico*
}

\author{
Drs. JUAN C. BAHAMONDES S. ${ }^{1}$, PABLO SANDOVAL M. ${ }^{2}$, HÉCTOR LOSADA M. ${ }^{2}$, GUSTAVO MERIÑO S. ${ }^{1}$, \\ JUAN SALMAN A. ${ }^{1}$, ABELARDO SILVA V. ${ }^{1}$, JAVIER MORA P. ${ }^{2}$ \\ 1 Centro Cardiovascular Hospital Regional. \\ 2 Clínica Alemana. \\ Departamento de Cirugía. Facultad de Medicina. Universidad de La Frontera. \\ Temuco, Chile.
}

\begin{abstract}
Hepatic hydatid cyst invading inferior vena cava with embolic membranes to right atrium. Report of one case
\end{abstract}

We report a 30 year old female admitted with a story of right upper quadrant pain and previous hepatic surgery for hydatid disease. A thoracoabdominal angio CAT scan and transesophageal echocardiogram revealed a mass located in the right atrium. The patient was operated and the right atrial mass was excised. Pathological study of the surgical piece confirmed a hydatid cyst. Post operative recovery was uneventful.

Key words: Hydatid cyst, atrial mass, cardiac surgery.

\section{Resumen}

La hidatidosis es una zoonosis parasitaria, de mayor incidencia en el sur de Chile. Su localización más frecuente es hepática y pulmonar. Sus complicaciones derivan de su crecimiento y afección de estructuras adyacentes o de su ruptura a cavidades. Presentamos un caso de quiste hidatídico hepático complicado, en una paciente que debutó con cuadro de dolor abdominal localizado en hipocondrio derecho producto de una lesión quística hepática con comunicación a vena cava inferior, siendo intervenida en su hospital de origen. Posteriormente AngioTAC y ecocardiografía transesofágica confirmaron masa intra auricular. Se realizó extracción de la masa bajo paro circulatorio con hipotermia profunda, correspondía a membranas hidatídicas y trombos adheridos a ella. La paciente evolucionó satisfactoriamente.

Palabras clave: Procedimientos quirúrgicos cardíacos $(\mathrm{MeSH})$, Heart valves $(\mathrm{MeSH})$, válvulas cardíacas $(\mathrm{MeSH})$, tumores cardíacos $(\mathrm{MeSH})$.

*Recibido el 10 de Marzo de 2009 y aceptado para publicación el 24 de Noviembre de 2009.

Este estudio no tuvo apoyo financiero.

Correspondencia: Dr. Juan Carlos Bahamondes S.

Av. Manuel Montt $112.4^{\circ}$ piso. Temuco, Chile.

Fax: 245325760

E-mail: jcbahamo@ufro.cl 


\section{Introducción}

La hidatidosis es una zoonosis endémica en el sur de Chile, sobretodo en el ámbito rural, producida por la larva del Echinococcus granulosus. Tiene una distribución mundial, sin embargo, la mitad sur de Sudamérica, Islandia, Australia, Nueva Zelandia y las regiones del sur de África tienen una elevada incidencia ${ }^{1}$. En nuestro país, las regiones con mayor actividad agrícola, en las cuales se cohabita con el huésped definitivo (canes), e intermediarios como ovinos, bovinos, porcinos caprinos, etc. son las que tienen la mayor prevalencia reportada ${ }^{2,3}$. Desde 1951 constituye una enfermedad de notificación obligatoria, sin embargo, se sospecha su subnotificación.

La infección en los humanos es accidental tras el contacto directo con los perros. El huevo se ingiere y al llegar al intestino ingresa a la circulación portal. Una vez en la sangre, son el hígado y el pulmón los órganos encargados de actuar como filtro hacia el resto del cuerpo, siendo estas las localizaciones más frecuentes ${ }^{4}$. Menos de un $10 \%$ de los parásitos logra evadir los filtros y adquirir otra localización como cerebro, riñones, bazo, páncreas, corazón, o bien, llegar a localizaciones inusuales ${ }^{5-8}$.

En el presente reporte, se presenta un caso de afección inusual por hidatidosis. Un quiste hepático que se complicó con comunicación a vena cava inferior (VCI) y embolización de membranas hidatídicas hacia aurícula derecha (AD). Cabe destacar que esta posibilidad, es uno de los diagnósticos diferenciales que se deben plantear ante el hallazgo de una masa intracardíaca. Se describe que en la hidatidosis el compromiso cardíaco es de baja frecuencia $(0,5-$ $2 \%)^{9}$ y de regla se asocia a otras localizaciones (hepática y pulmonar). Excepcionales son los reportes de afectación cardíaca aislada ${ }^{10}$.

\section{Caso clínico}

Mujer de 30 años de edad, residente en la comuna de Castro, X Región de Los Lagos, sin antecedentes de importancia en su historia clínica. Consultó en su Hospital por cuadro de una semana de evolución, caracterizado por compromiso del estado general, tos irritativa de predominio nocturno y expectoración mucosa, por lo cual se indican antibióticos. A los dos días siguientes sufre episodio descrito como síncope, en el cual también habría presentado convulsiones. Fue interpretado en el contexto de epilepsia, estudiándose como tal, sin embargo, exámenes (electroencefalograma y TAC de cerebro) no presentaron alteraciones. Continuó recibiendo antibióticos por cuadro infeccioso de origen respiratorio. Al completar cinco días de tratamiento, inicia fiebre de hasta $39^{\circ} \mathrm{C}$ y dolor abdominal difuso, de mayor intensidad en hipocondrio derecho que la obliga a consultar nuevamente. Se realiza ecografía abdominal, en la cual se pesquisa gran lesión quística del lóbulo hepático derecho, razón por la cual se traslada ese mismo día al Hospital de Puerto Montt. En este centro se realiza TAC de abdomen, que muestra imagen quística hepática que se comunica a VCI, con una masa en su interior extendida a AD. Seguidamente, se efectúa ecocardiograma informado con hallazgos de masa en AD concordante con tumor intra auricular o trombo.

A los cuatro días de hospitalizada se realiza cirugía de quiste hidatídico hepático por laparotomía subcostal. Protocolo operatorio describe hígado con gran aumento de tamaño y descendido. Lesión quística en lóbulo derecho que se punciona extrayendo $240 \mathrm{cc}$. de líquido hemático. Se extraen membranas hidatídicas tras lo cual se produce un sangrado masivo que se logra controlar ampliando el acceso a la lesión, identificando luego un orificio de comunicación con VCI de $1,8 \mathrm{~cm}$ de diámetro aproximadamente. Se extraen membranas hidatídicas desde el orificio comunicante, suturándose el vaso posteriormente. Se reseca pared quística y se sutura hígado tras quistectomía. Además se practica colecistectomía.

Tras la cirugía, la paciente requiere de reposición de glóbulos rojos, tratamiento antibiótico y manejo en unidad de cuidados intensivos. Persiste masa intracardíaca a pesar de la extracción de membranas hidatídicas desde orificio comunicante a VCI.

A los dos días del postoperatorio, la paciente se traslada a Clínica Alemana de Temuco con los siguientes diagnósticos: Quiste hidatídico hepático comunicado a VCI y embolización de membranas hidatídicas a $\mathrm{AD}$, sepsis de foco abdominal, segundo día post quistectomía, extracción de membranas hidatídicas y sutura de VCI. En este centro asistencial se realiza ecocardiografía transesofágica (ETE) que muestra imagen ecogénica adherida a la pared de VCI, de implantación fija de 2 por $1 \mathrm{~cm}$ a $2,5 \mathrm{~cm}$ de la llegada a $\mathrm{AD}$ que se continúa con una porción móvil que se extiende hacia el interior de ella. El flujo sanguíneo en la vena cava inferior es normal. Demás cavidades y válvulas cardíacas de aspecto normal. Se concluye que probable etiología es un trombo.

Además, se practica angio TAC de tórax que muestra defecto hipodenso aproximadamente de 2 por $0,9 \mathrm{~cm}$ altamente sugerente de trombo, que se inicia en segmento suprahepático derecho, que altera el llenado de la VCI, y que se extiende a AD. Resto del examen no muestra alteraciones patológicas en pulmón ni corazón izquierdo.

Dados los antecedentes clínicos previos de la paciente, se presume que la masa corresponde a 
membranas hidatídicas implantadas en la pared de la VCI flotantes hacia AD donde existe inminente riesgo de embolización, motivo por el cual se decide programar cirugía cardíaca para extraer los restos hidatídicos.

Se accede al corazón a través de esternotomía media. Se utiliza circulación extracorpórea mediante canulación en cayado aórtico proximal y retorno venoso con canulación bicava. Una vez alcanzados los $22^{\circ} \mathrm{C}$, se efectúa el paro cardiocirculatorio y perfusión cerebral retrógrada por vena cava superior durante este período (18 min). A través de auriculotomía derecha y retirando momentáneamente la cánula venosa en VCI durante el paro circulatorio se realiza extracción de masa intracardíaca en su totalidad, luego de lo cual se procedió al recalentamiento sistémico de la paciente reiniciando la circulación extracorpórea y luego reasumiendo el corazón su ritmo sinusal previo. La cirugía fue llevada a cabo sin incidentes. Biopsia contemporánea del material demostró restos de membranas hidatídicas y trombos adheridos.

Paciente requiere manejo en unidad de cuidados intensivos, tratamiento antibiótico y reposición de glóbulos rojos. Evoluciona satisfactoriamente, trasladándose a los siete días de la operación a su hospital base.

\section{Discusión}

Más del $90 \%$ de los quistes hidatídicos se ubican en hígado y pulmón ${ }^{11}$. El 10\% restante, logra pasar estos filtros biológicos, por medio de anastomosis porto-cava o por circulación linfática a través del conducto torácico ${ }^{12}$, alojándose en lugares inusuales como el cerebro, riñón, mediastino y corazón, entre otras ubicaciones ${ }^{13,14}$. El quiste hidatídico es muchas veces un hallazgo incidental, pudiendo manifestar síntomas dados por su crecimiento, los que dependen de su localización y estructuras afectadas, rara vez se manifiesta como un síndrome anafiláctico producto de su ruptura.

La enfermedad hidatídica hepática presenta comúnmente dolor en hipocondrio derecho y hepatomegalia. Entre las manifestaciones extraabdominales se describen síntomas respiratorios y síndrome febril inespecífico, como se evidenció en la historia de nuestra paciente ${ }^{15}$.

Se puede recurrirr para el diagnóstico a algunos exámenes serológicos o pruebas cutáneas, los que tienen una sensibilidad y especificidad bajas en estos $\operatorname{casos}^{13,14}$.

La complicación de un quiste hidatídico hepático puede ser su ruptura causando siembra a peritoneo o shock anafiláctico ${ }^{1,3,9,11}$. Las comunicaciones pueden estar en el contexto de su tránsito hepato-torácico pasando por las estructuras adyacentes a esta localización, donde se encuentra la VCI, espacio pleural y bronquios ${ }^{14,16,17}$. Nuestra paciente no presentó biliptisis, aunque eventualmente se podría haber sospechado esta complicación tras el hallazgo del quiste, ante la tos y expectoración mucosa que se estaba tratando. Claramente no había evidente compromiso de la función respiratoria.

La comunicación del quiste abre la posibilidad que la enfermedad se disemine a otros órganos, lo que se descartó a priori en este caso clínico, sin embargo, requiere de un seguimiento a largo plazo, y exámenes serológicos que confirmen la erradicación del parásito. Para ello, el manejo con Albendazol es una opción.

El hallazgo de una masa intracardíaca nos obliga a realizar los diagnósticos diferenciales, que corresponden en orden decreciente, a trombos, tumores (mixomas o metástasis), vegetaciones dependientes de válvulas protésicas, o nativas en endocarditis, $u$ otras posibilidades como variantes normales (red de Chiari, válvulas de Eustaquio, falsos tendones, bandas moderadoras, aneurismas del septo interauricular y excrecencias de Lambl) cuya localización y características ecocardiográficas las hacen fácilmente identificables ${ }^{1,7,9,11,17}$. La posibilidad de otra etiología, se reduce a la historia clínica previa del paciente y los descubrimientos imagenológicos, como en el caso presentado.

La historia de síncope y convulsiones al inicio del cuadro clínico, hace sospechar la posibilidad de una alteración del ritmo con caída del gasto cardíaco, que podría relacionarse con el contacto directo de la masa intracardíaca con tejido excito-conductor o con la afectación momentánea del retorno venoso al corazón derecho.

La mortalidad de la hidatidosis es baja, sin embargo, repercute social y económicamente en los servicios de salud, por ser una enfermedad totalmente prevenible, que ocupa recursos importantes. Por esta razón, en sitios endémicos, se debe tener un alto índice de sospecha para detectar esta enfermedad antes de que presente secuelas sistémicas.

\section{Referencias}

1. Schwartz. Principios de Cirugía, volumen II, $7^{\circ}$ edición, México, Interamericana, 2000: 1497-1498.

2. Aliaga F, Oberg C. Epidemiología de la hidatidosis humana en la IX Región de la Araucanía, Chile 19911998. Bol Chil Parasitol 2000; 55: 3-4.

3. Schenone H, Contreras M, Salinas P, Sandoval L, Saavedra T, Rojas A. Hidatidosis humana en Chile. Seroprevalencia y estimación del número de personas 
infectadas. Bol Chil Parasitol 1999; 54: 3-4.

4. Engin G, Acunas B. Hydatid disease with inusual localization. Eur Radiol 2000; 10: 1904-1912.

5. Pedrero C, San Juan S, Lozano J L, Morán J. Quiste hidatídico en mediastino. Cir Pediatr 2001; 14: 127-128.

6. Heras F, Ramos G, Duque J, García M, Cerezuela J, Matilla J. Quistes hidatídicos de mediastino: 8 casos. Arch Bronconeumol 2000; 36: 221-224.

7. Miranda R, Merchak A, Ferrier P, Villarroel A, Edding O. Quiste hidatídico cardiopericárdico: presentación de dos casos clínicos. Rev Chil Radiol 2002; 8: 123 126.

8. Álvarez R, Alarcón E, González R. Quiste hidatídico retroperitoneal. Rev Chil Cir 2002: 54; 536-538.

9. Dighiero J, Canabal EJ. Echinococcus disease of the heart. Circulation 1958; 17: 127-132.

10. Laglera S, García MA, Martínez F. A case of cardiac hydatidosis. Br J Anaesth 1997; 79: 671-673.

11. Salman J, Meriño G, Silva A, Bahamondes J, Cherres O,
Horta J. Hidatidosis intracardíaca. Rev Chil Cir 2000; 52: 285-290.

12. Pérez JA, Pérez A, Valenzuela M. Quistes hidatídicos primitivos de localización extraabdominal y extrapulmonar. Rev Chil Cir 1999; 51: 341-346.

13. Kuschel C, Aho A, Ojeda V. Hidatidosis Hepática: Experiencia quirúrgica en Hospital Regional de Valdivia, noviembre 1988 - octubre 2003. Revista de Estudiantes de Medicina del Sur 2003; 2: 53-58.

14. Eroglu A, Kurkcuoglu N. Primary hydatid cysts of the mediastinum. Eur J Cardiothorac Surg 2002; 22: 599-601.

15. Losada H, Vial M, Manterola C. Fístula biliobronquial secundaria a quiste hepático hidatídico en tránsito al tórax: Reporte de caso. Rev Chil Cir 2006; 58: 224-227.

16. De la Torre J, Mayayo E, Fernández F. Varón con masa intracardíaca e insuficiencia cardíaca. Rev Esp Patol 2003; 36: 339-346.

17. Little J. Recurrence of hydatid disease. World J Surg 1988; 12: 700-704. 